\title{
Una intervención en aprendizaje cooperativo sobre el perfil del observador en la dinámica bullying*
}

\section{Intervention effectiveness in cooperative learning on the profile of} the dynamics bullying observer

Recepción: 17 Octubre 2013 | Aprobación: 23 Enero 2017

\author{
M Isabel Polo del Río ${ }^{\mathrm{a}}$ \\ Universidad de Extremadura, España \\ ORCID: http://orcid.org/0000-0001-7107-1330 \\ Santiago Mendo Lázaro \\ Universidad de Extremadura, España \\ Fernando Fajardo Bullón \\ Universidad de Extremadura, España \\ Benito León del Barco \\ Universidad de Extremadura, España
}

a Autor de correspondencia. Correo electrónico:
mabelpdrio@unex.es

Para citar este artículo: Polo del Río, M.I., Mendo Lázaro, S., Fajardo Bullón, F. \& León del Barco, B. (2017). Una intervención en aprendizaje cooperativo sobre el perfil del observador en la dinámica bullying. Universitas Psychologica, 16(1), 1-13, http://dx.doi.org /10.11144/Javeriana.upsy16-1.iacp

\section{RESUMEN}

El bullying (acoso escolar) contempla como protagonistas a un grupo de alumnos con papeles bien diferenciados: Agresor, víctima y observadores, estos últimos, testigos del acoso que pueden condicionar el curso del mismo. Con este estudio se pretende determinar si la aplicación de técnicas de aprendizaje cooperativo en el aula, reduce la frecuencia de conductas de acoso escolar, en estudiantes de Educación Primaria. Con el fin de evaluar dichas conductas, se aplicó la Escala de frecuencias de acoso escolar entre iguales. Perspectiva Observador. Nuestros resultados apoyan la hipótesis, de que tras la aplicación de técnicas de aprendizaje cooperativo en el aula, los observadores constatan una reducción de las agresiones verbales y físicas directas, físicas indirectas y de exclusión social propias de la dinámica bullying.

Palabras clave

bullying; observador; aprendizaje cooperativo.

\begin{abstract}
Bullying includes starring a group of students with distinct roles: aggressor, victim and observers, the latter witnessed the harassment, which may affect the course of the same. This study aims to determine whether the application in the classroom cooperative learning techniques, reduces the frequency of bullying behaviors in students of Primary Education. In order to evaluate these behaviors, the Frequency Scale Peer Bullying. Observer Perspective applies. Our results support the hypothesis that following the implementation of cooperative learning techniques in the classroom, observers establish that reduction in direct verbal and physical aggression, indirect and dynamic characteristics of social exclusion physical bullying. Keywords

bullying; observer; cooperative learning.
\end{abstract}


Maa Isabel Polo del Río, Santiago Mendo Lázaro, Fernando Fajardo Bullón, Et al.

\section{Introducción}

El acoso escolar es una forma específica de maltrato entre iguales, conocida por el vocablo inglés bullying (Olweus, 1983), que se caracteriza por la agresión de uno o varios alumnos, hacia otro de forma intencionada, existiendo un desequilibrio de poder (abuso) por parte del primero hacia el segundo. Dicho fenómeno ha sido observado en todos los países en los que ha sido estudiado, provocando en las víctimas efectos claramente negativos: descenso de la autoestima, ansiedad o depresión, retraimiento social (Polo et al., 2015), lo que dificulta su integración en el medio escolar y el desarrollo normal de los aprendizajes (León, Gozalo, \& Polo, 2012).

El bullying tiene como protagonistas a un grupo de alumnos con papeles bien diferenciados (Avilés, 2004; Cerezo, 2014). Entre ellos están la víctima y el agresor: la víctima, sujeto que recibe las agresiones de otro, de manera sistemática (Mendoza, Morales, \& Arriaga, 2015), vive y sufre la situación con tal ansiedad que puede llegar a padecer graves estados de ansiedad o depresivos, aislamiento social o baja autoestima (Polo et al., 2015), y el agresor cuyo comportamiento se basa en el uso de poder de forma agresiva sobre la víctima (Gutiérrez, Benítez, Machado, \& Justicia, 2012).

Los observadores son todos aquellos alumnos, que contemplan la situación sin intervenir (Avilés \& Monjas, 2005; Ruíz, Riuró, \& Tesouro, 2014), guardando silencio como espectadores pasivos del acoso por parte del agresor (Cerezo \& Sánchez, 2013), no cuestionan para nada su comportamiento por temor a convertirse en víctimas (Trautmann, 2008), o porque se sienten al margen del problema (Molina \& Vecina, 2015). Mientras que en otras ocasiones, intervienen bien a favor de la víctima, realizando acciones para detener los comportamientos del agresor, o bien instigando, alentando, y apoyando al agresor, y contribuyendo al acoso escolar con actos intimidatorios (Cuevas \& Marmolejo, 2016). El hecho de observar actitudes agresivas entre compañeros, produce en ocasiones, un contagio social que da lugar a la participación (Cerezo, 2006).

La intervención de los observadores como participantes activos puede condicionar de alguna manera el curso del acoso escolar, de tal manera que si apoyan a la víctima se anula el acoso, mientras que si el apoyo se lo dan al agresor se produce un refuerzo del mismo (Cuevas \& Marmolejo, 2016).

En relación con el género y la edad, los chicos asumen con frecuencia roles pro-bullying (Haro \& García, 2014) convirtiéndose en observadores pasivos según avanza su edad, mientras que no parece suceder lo mismo en las chicas (Caballo, Calderero, Arias, Salazar, \& Irurtia, 2012), que son consideradas más defensoras y espectadoras (Hoyos, Llanos, \& Valega, 2012; Haro \& García, 2014). En el Informe del Defensor del pueblo (2006) se encuentran diferencias en función del curso y el género de los estudiantes, en cuanto a las observaciones de conductas de agresión propias de la dinámica bullying, por lo que resulta relevante el análisis de la eficacia de la intervención en aprendizaje cooperativo, considerando dichas variables.

De esta manera, el aprendizaje cooperativo es una metodología esencial para mejorar las relaciones y la convivencia en el ámbito educativo (Antolín, Martín-Pérez, \& Barbá, 2012; León, Polo, Gozalo, \& Mendo, 2016), y es considerada como una de las acciones de intervención centrales para luchar contra el acoso escolar (Olweus, 1993; Avilés, Irurtia, García-López, \& Caballo, 2011).

La mayoría de las investigaciones sobre aprendizaje cooperativo, se han centrado en analizar las consecuencias y los resultados de la aplicación de técnicas de aprendizaje cooperativo en el aula sobre variables académicas, sociales y afectivas (Johnson, Johnson, \& Maruyama, 1983; Pérez-Sánchez \& Poveda, 2008; León, Mendo, Felipe, Polo, \& Fajardo, 2017). Las investigaciones realizadas concretamente sobre los efectos del aprendizaje cooperativo en variables sociales, desde hace casi cuatro décadas, han encontrado que estas técnicas mejoran las relaciones humanas (Devries \& Edwards, 1974; Cook, 1978; Slavin, 1978; Ovejero, 1990; 
Smith, Boulton, \& Cowie, 1993; Slavin \& Cooper, 1999; Goikoetxea \& Pascual, 2002; DíazAguado, 2003; Pérez-Sánchez \& Poveda, 2008; León et al., 2012; Vega, Vidal, \& García, 2013).

Las consecuencias que genera la metodología de aprendizaje cooperativo para los alumnos en el aula son positivas y muy beneficiosas, puesto que proporciona aquellas estrategias básicas necesarias para desarrollar interacciones sociales saludables, destacando entre ellas, un aumento del apoyo social, mayor atracción interpersonal entre los alumnos y actitudes más positivas hacia los demás (Ovejero, 1990; Johnson \& Johnson, 1990; Pedreira \& González-Fontao, 2014), consecuencias todas ellas contrarias a la aparición de las conductas propias del acoso escolar (Johnson \& Johnson, 2000). Además, las técnicas de aprendizaje cooperativo constituyen un método adecuado para adquirir habilidades y competencias sociales necesarias en la prevención e intervención sobre el problema del acoso escolar (León, Gozalo, \& Vicente, 2004; León, Felipe, Mendo, \& Iglesias, 2015) y desarrollan la empatía o capacidad del sujeto para ponerse en circunstancias distintas a la propia (Johnson, 1975; Moriña, 2011).

El acoso escolar es, por tanto, un fenómeno en el que agresor, víctima y observadores, son partes integrantes de un mismo proceso. Está clasificado como uno de los problemas de convivencia que existen actualmente en los centros escolares (Ortega, Del Rey, \& Casas, 2013). Por ello, se hace necesario atajar el problema en el mismo entorno en que se produce (López, Bilbao, \& Rodríguez, 2012; Ramírez-Pavelic, 2013), a través de distintas intervenciones, dirigidas a todos los alumnos del aula.

El aprendizaje cooperativo puede ser una solución que posibilite la creación de relaciones de alta calidad entre iguales y proporcione a los alumnos esas estrategias básicas necesarias, para desarrollar unas adecuadas interacciones interpersonales. Es importante analizar la eficacia del aprendizaje cooperativo sobre la dinámica bullying, no solo desde la perspectiva del agresor o la víctima, sino también desde la perspectiva del observador. Un mismo caso de agresión puede ser observado por varios testigos, lo que supone un motivo de preocupación, ya que la observación de la violencia es un elemento fundamental, a través del cual niños y adolescentes aprenden y desarrollan conductas agresivas (León, Felipe, Gómez, \& López, 2011). Así pues, con base en la investigación previa, en este estudio se pretende determinar si desde la perspectiva de los observadores, la aplicación de técnicas de aprendizaje cooperativo en el aula reduce la frecuencia de conductas de acoso escolar, en estudiantes de Educación Primaria.

\section{Método}

\section{Participantes}

La muestra estuvo formada por 110 estudiantes de Educación Primaria, el 52.7 \% niñas y 47.3 $\%$ niños, de edades comprendidas entre los 10 y $\operatorname{los} 12$ años $(M=11.76, D T=0.72)$. De quinto curso de primaria hubo 68 alumnos y 42 fueron de sexto de primaria. Los centros fueron elegidos de forma aleatoria por conglomerados. Se seleccionaron al azar tres centros educativos públicos de la provincia de Cáceres (España). Uno de los centros fue urbano (2 grupos de $5^{\circ}$ de primaria y dos grupos de $6^{\mathrm{O}}$ de primaria) y dos fueron rurales (Un grupo de 5o de primaria y $6^{\mathrm{O}}$ en cada centro). El procedimiento empleado consistió en asignar números a todos los colegios que aceptaron participar en el estudio y a través de números aleatorios generados por ordenador elegir los centros que finalmente participaron.

\section{Instrumentos}

El instrumento utilizado fue la Escala de frecuencias de acoso escolar entre iguales. Perspectiva Observador para medir la frecuencia de las conductas de agresión propias de la dinámica bullying, desde una de las perspectivas del acoso entre iguales: la perspectiva del observador. La escala consta de 16 ítems, y se presenta en formato Likert con cinco intervalos en forma numérica de 1 al 5, que representan un continuo que va desde "Nunca" hasta 
"Siempre". Estos ítems muestran las conductas de agresión características en las situaciones de acoso escolar. Siguiendo la definición clásica de Farrington (1993) y los trabajos de Del Barrio, Martín, Montero, Fernández y Gutiérrez (2003) se consideran conductas de maltrato, directas (físicas y verbales) e indirectas, así como conductas de exclusión social.

La escala evalúa 4 factores: El primer factor "Agresiones verbales y físicas directas", incluye 6 ítems y se refiere a conductas observadas como: hablar mal, despreciar, burlarse, amenazar, pegar, ridiculizar a compañeros. El segundo factor "Agresiones físicas indirectas", incluye 4 ítems y se refiere a conductas observadas como: empujar a otros compañeros, y lanzar, tirar, y destrozar objetos de otros compañeros. El tercer factor "Agresiones de exclusión social", incluye 4 ítems y se refiere a conductas observadas, como: no dejar participar en un juego, dejar solos, rechazar e ignorar a otros compañeros. El cuarto factor "Agresiones verbales in-directas" incluye 2 ítems y se refiere a conductas observadas como: contar historias falsas y chantajear a otros compañeros.

La consistencia interna de la escala, medida a través del índice Alfa de Cronbach, indica una excelente fiabilidad global $(\alpha=0.939)$, buena para los factores $1(\alpha=0.884), 2(\alpha=0.832)$ y $3(\alpha=0.843)$ y aceptable para el factor $4(\alpha=$ 0.688).

\section{Diseño}

Se utilizó una metodología cuasi-experimental, con un diseño intra e intergrupo pretest-postest con grupo control no equivalente. Los grupos se formaron naturalmente, no al azar. Las dos estrategias fundamentales para paliar los defectos de la metodología cuasi-experimental fueron: 1 . La inclusión de un grupo control. 2. Tomar una medida tras la aplicación del tratamiento y otra previa al mismo. A estas dos medidas se les denomina postest y pretest.

Se ha trabajado con grupos independientes, un grupo experimental, formado por 45 alumnos de $5^{\circ}$ de primaria y 13 alumnos de $6^{\circ}$ de primaria distribuidos en cuatro aulas pertenecientes a tres centros y un grupo control, formado por 23 alumnos de $5^{\text {o }}$ de primaria y 29 de $6^{0}$ de primaria distribuidos, igualmente, en cuatro aulas de los centros participantes. Existe una condición experimental, la intervención con técnicas de aprendizaje cooperativo, concretamente la técnica de Jigsaw. El grupo control no recibe la intervención y ambos grupos, experimental y control, cumplimentan en fase pretest y postest la Escala de Frecuencia de Acoso Escolar entre Iguales. Perspectiva Observador.

\section{Procedimiento}

A los profesores que voluntariamente quisieron participar en la experiencia se les entrenó en un seminario de doce horas en aprendizaje cooperativo. La intervención en técnicas de aprendizaje cooperativo se desarrolló durante cuatro meses.

La técnica utilizada es la de Jigsaw o técnica de Rompecabezas o Mosaico, en esta técnica el tema objeto de aprendizaje, se divide en tantas partes o fragmentos como miembros tiene el equipo. Cada uno de los alumnos recibe un trozo del tema que tiene que leer, estudiar y preparar. Cuando estén seguros de dominar el material, vuelven al grupo y enseñan al resto de compañeros lo que han aprendido. Entre todos se intenta resolver dudas, aclarar, preguntar, explicar... con el objetivo de aprender todo el material. El motivo que nos lleva a elegir esta técnica se debe, en palabras de Aronson \& Osherow (1980), a que su aplicación incrementa la atracción de los estudiantes hacia sus compañeros y hacia la institución escolar, disminuye la competitividad y aumenta la capacidad para ponerse en el lugar o papel de otra persona, todos ellos, factores necesarios para reducir las conductas de acoso escolar en el aula y fuera de ella.

\section{Análisis estadísticos}

En primer lugar se sometieron los datos a la prueba de Kolmogorov-Smirnov, para comprobar si se cumple el supuesto de normalidad. La distribución es normal en los factores 1 
(Observar Agresiones Verbales y físicas directas), 2 (Observar agresiones físicas indirectas) y 3 (Observar agresiones de exclusión social), utilizando pruebas paramétricas en estos factores. En el factor 4 (Observar agresiones verbales indirectas) la distribución no cumple el supuesto de normalidad, por lo que se utilizan pruebas y no paramétricas.

Posteriormente, para contrastar si la intervención realizada reduce la puntuación obtenida en la Escala de Frecuencia de Acoso Escolar entre Iguales. Perspectiva Observador, se realizan comparaciones intragrupo (t de Student y Rangos de signos de Wilcoxon) e intergrupos ( $t$ de Student, y la U de Mann-Whitney). Además, para determinar la eficacia de la intervención, completando la información que aporta la aplicación de las pruebas de significación se calcula el tamaño del efecto, utilizando el estadístico d, propuesto por Cohen (1977), ya que, en ocasiones un resultado "no significativo" puede tener, una significación práctica (Kirk, 1996). De esta forma, la consideración de la magnitud del efecto es utilizada para determinar si los resultados son irrelevantes o, útiles e importantes.

Por último, para reducir el grado de error experimental y determinar la eficacia de la intervención se realizaron análisis de la covarianza (ANCOVA) utilizando como factores fijos el grupo (experimental/control), el curso (5은 y 6o de primaria) y el género.

\section{Resultados}

En primer lugar, se presentan los resultados de las pruebas t de Student y Rangos de signos de Wilcoxon para muestras relacionadas (Tabla 1). En este sentido, las comparaciones intragrupo experimental muestran una disminución $(p \leq$ 0.05) entre las puntuaciones pretest y postest, con tamaños del efecto pequeños $(d<0.30)$ en los factores 2 (Observar agresiones físicas) y 3 (Observar agresiones de exclusión social). En cuanto a los resultados obtenidos en las pruebas intragrupo control, como se puede observar en la Tabla 1, no se encuentran diferencias significativas en ninguna de las modalidades de agresión.

\section{TABLA 1}

Diferencias de medias intragrupo y tamaño del efecto de la intervención en técnicas de aprendizaje cooperativo

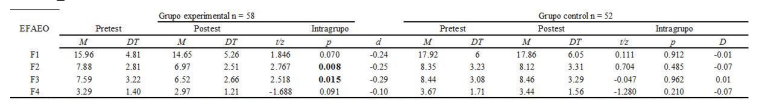

Nota: EFAEO = Escala de Frecuencia de Acoso Escolar entre Iguales. Perspectiva Observador; Factores de EFAEO: F1 = Agresiones Verbales y físicas directas; F2 = Agresiones físicas indirectas; F3 = Agresiones de exclusión social; F4 =

Agresiones verbales indirectas. $t=\mathrm{t}$ de Student; $z$ $=$ Prueba de Rangos de Wilcoxon, $d=\mathrm{d}$ de Cohen Fuente: elaboración propia.

En cuanto a las comparaciones intergrupo (control/experimental) (Tabla 2), sólo se encuentra diferencias significativas en las comparaciones postest. Los datos muestran que los sujetos del grupo experimental obtienen puntuaciones $(p \leq 0.005)$ menores en los factores 1 (Observar agresiones verbales y físicas directas), 2 (Observar agresiones físicas indirectas) y 3 (Observar agresiones de exclusión social) y tamaños del efectos de una magnitud media $(d>0.40)$.

\section{TABLA 2}

Diferencias de medias intergrupos, tamaño del efecto y eficacia de la intervención en técnicas de aprendizaje cooperativo

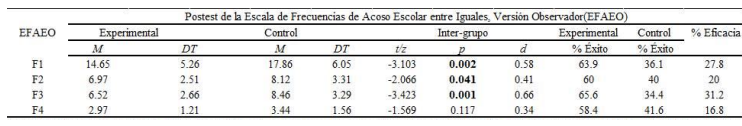

Nota: EFAEO = Escala de Frecuencia de Acoso Escolar entre Iguales. Perspectiva Observador; Factores de EFAEO: F1 = Agresiones Verbales y físicas directas; F2 = Agresiones físicas indirectas; F3 = Agresiones de exclusión social; $\mathrm{F} 4=$ Agresiones verbales indirectas. $t=\mathrm{t}$ de Student; $z$ = Prueba de Mann-Whitney, $d=d$ de Cohen. \%Éxito = binomial effect size display. Fuente: elaboración propia.

Además, con el objetivo de interpretar la efectividad de la intervención se calcula el BESD (Binomial Effect Size Display) que permite confeccionar una tabla de éxitos (Tabla 2).

Con respecto al factor Observar agresiones verbales y físicas directas, para el grupo 
experimental se obtiene un porcentaje de éxito de $63.9 \%$ y un $36.1 \%$ para el grupo control. En definitiva, el grupo que recibió la intervención consiguió un $27.8 \%$ más de eficacia que el grupo control, en este factor. Con respecto al factor Observar agresiones físicas indirectas, para el grupo experimental se obtuvo un porcentaje de éxito de $60 \%$ y un $40 \%$ para el grupo control. Por tanto, el grupo que recibió la intervención consiguió un $20 \%$ más de eficacia que el grupo control sobre dicho factor. Con respecto al factor Observar agresión de exclusión social, para el grupo experimental se obtuvo un porcentaje de éxito de $65.6 \%$ y un $34.4 \%$ para el grupo control. Esto indica, que el grupo que recibió la intervención consiguió un $31.2 \%$ más de eficacia que el grupo control, en dicho factor. Por último, el factor Observar agresiones verbales indirectas, para el grupo experimental se obtuvo un porcentaje de éxito de $58.4 \%$ y un $41.6 \%$ para el grupo control, siendo el factor donde el porcentaje de eficacia del grupo experimental es menor: $16.8 \%$.

Finalmente, para eliminar de las variables dependientes (puntuaciones postest de la EFAEO) el efecto atribuible a variables no incluidas en el diseño y por tanto, no sometidas a control experimental, y como complemento a las pruebas intra e intergrupos, se realizan pruebas de efectos intersujetos (ANCOVA) en función del grupo de intervención, el curso y el género.

Por un lado, en la Tabla 3 se utilizan como covariables las puntuaciones pretest de las variables dependientes (EFAEO) y como factor fijo los grupos de intervención (experimental y control). De esta manera, se confirma si las diferencias estadísticas anteriormente mostradas en las Tablas 1 y 2 pueden ser atribuidas a la intervención.

\section{TABLA 3}

Prueba de efectos intersujetos (ANCOVA) en la Escala de Frecuencias de Acoso Escolar entre Iguales, Versión Observador (EFAEO), factor fijo grupo experimental/control

\begin{tabular}{lccccc} 
VD: Factores EFAEO (postest) & \multicolumn{5}{c}{ Intervención } \\
\cline { 2 - 6 } Origen: Factores EFAEO (pretest) & gl.(error) & RMS & $F$ & $p$ & $\eta^{2}$ \\
\hline F1. Agresiones verbales y fisicas directas & $1(107)$ & 95.500 & 4.975 & $\mathbf{0 . 0 2 8}$ & 0.045 \\
F2. Agresiones fisicas indirectas & $1(107)$ & 19.779 & 4.091 & $\mathbf{0 . 0 4 6}$ & 0.037 \\
F3. Agresiones de exclusión social & $1(107)$ & 64.349 & 9.523 & $\mathbf{0 . 0 0 3}$ & 0.082 \\
F4.Agresiones verbales indirectas & $1(107)$ & 3.340 & 1.981 & 0.162 & 0.018 \\
\hline
\end{tabular}

Fuente: elaboración propia.
El ANCOVA muestra diferencias significativas $p<0.05$ entre los grupos (experimental y control) en los factores 1,2 y 3 de la EFAEO. No se encontraron diferencias en el factor 4 que puedan atribuirse a la intervención. De esta forma se confirma la reducción de las observaciones en el grupo experimental de las agresiones verbales y físicas directas, físicas indirectas y de exclusión social tras la intervención.

Por otro lado, para conocer el efecto del curso y el género en la intervención, en la Tabla 4 se utilizan como covariables las puntuaciones pretest de las variables dependientes (EFAEO) y como factores fijos el curso y género.

\section{TABLA 4}

Prueba de efectos intersujetos (ANCOVA) del grupo experimental, en la Escala de Frecuencias de Acoso Escolar entre Iguales, Versión Observador (EFAEO), factores fijos curso y género

\begin{tabular}{|c|c|c|c|c|c|c|c|c|c|}
\hline \multirow{2}{*}{ 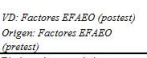 } & \multirow{2}{*}{$\begin{array}{l}\text { gl } \\
\text { (errot) }\end{array}$} & \multirow[b]{2}{*}{ RMS } & \multicolumn{2}{|c|}{ Curso } & \multirow[b]{2}{*}{$\eta^{2}$} & \multirow[b]{2}{*}{ RMS } & \multicolumn{2}{|c|}{ Género } & \multirow[b]{2}{*}{$n^{2}$} \\
\hline & & & $F$ & $p$ & & & $F$ & $p$ & \\
\hline 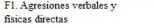 & 1(55) & 21906 & 0.949 & 0.334 & 0.20 & 62685 & 2.806 & 0.100 & 0.049 \\
\hline F2. Agres siones Fisicas indidrectas & 1(5) & 6.566 & 1.498 & 0.226 & 0.027 & 0.296 & 0.065 & 0.799 & 0.001 \\
\hline $\begin{array}{l}\text { F3. Agresiones de excclusion } \\
\text { social }\end{array}$ & $1(55)$ & 1.272 & 0.209 & 0.650 & 0.004 & 9.823 & 1.653 & 0204 & 0.029 \\
\hline 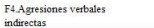 & $1(35)$ & 0.048 & 0.033 & 0.857 & 0.001 & 0.585 & 0.399 & 0.350 & 0.007 \\
\hline
\end{tabular}

Fuente: elaboración propia.

Como se puede apreciar en la Tabla 4, El ANCOVA no muestra diferencias $(\mathrm{p} \leq 0.05$ ) entre sujetos, ni en función del curso ni del género en ninguna de las comparaciones pretestpostest que puedan atribuirse a la intervención llevada a cabo.

\section{Discusión}

Los resultados apoyan la hipótesis de que tras la aplicación en el aula de técnicas de aprendizaje cooperativo, se reducirán las observaciones de conductas de agresión de unos compañeros hacia otros, concretamente en las modalidades de agresiones verbales y físicas directas, físicas indirectas y de exclusión social.

Estos son resultados coherentes con los que se obtuvieron en la investigación realizada sobre acoso escolar desde la perspectiva del agresor (León et al., 2012; León et al., 2016) en los que se expone que las agresiones de exclusión social son las conductas que se reducen en mayor 
medida, tras la intervención en aprendizaje cooperativo. De esta manera, se puede concluir que ciertamente los observadores han sido testigos de la reducción de conductas de agresión, tras la intervención en técnicas de aprendizaje cooperativo.

Los resultados que se obtienen coinciden con las conclusiones de Aronson \& Osherow (1980), las cuales indican que el método de aprendizaje cooperativo y en concreto la técnica de Jigsaw, producen un incremento en la atracción de los alumnos hacia sus compañeros (Llorent \& Varo, 2013), y por ende, una reducción en conductas de exclusión social. Para Díaz-Aguado (1996) el aprendizaje cooperativo contribuye a representar un procedimiento compensador de situaciones de privación social, al garantizar que todos los alumnos interactúan con sus compañeros de forma positiva (idea contraria a la exclusión social), de acuerdo con esto, Slavin (1992) destaca la perspectiva de cohesión social, según la cual los componentes de un grupo de alumnos que trabajan en aprendizaje cooperativo quieren ayudarse mutuamente, se interesan y preocupan unos de otros. Este hecho habrá favorecido el descenso de las modalidades de agresiones verbales y físicas directas, físicas indirectas, tal y como lo reflejan los datos aportados por los observadores, que manifiestan una reducción de conductas como "presenciar desprecios entre compañeros", "pegar a otros compañeros" y "tirar las cosas de otros compañeros", características de ambas modalidades.

Por otra parte, se confirma que el curso y el género son variables que no influyen en la eficacia de la intervención, en este sentido, Johnson, Johnson, y Holubec (1999) indican que el aprendizaje cooperativo es una metodología en la que variables como el género y la edad, son factores importantes para el docente, al organizar al alumnado en grupos heterogéneos

A partir de los resultados obtenidos en esta investigación, destacar por un lado, la importancia de la información que llega de los observadores, presentes en la mayoría de las situaciones en las que se producen conductas de agresión de unos compañeros hacia otros (DíazAguado, 2005); y por otro lado, los beneficios que reporta la intervención en aprendizaje cooperativo sobre el acoso escolar, ya que tras un período de aplicación de las técnicas de aprendizaje cooperativo en el aula, y a pesar de que las conductas de agresión son relativamente estables, tienden a perpetuarse en el tiempo y son resistentes al cambio (Trianes, Muñoz, \& Jiménez, 2007) el alumnado confirma haber sido testigo de una reducción de ciertas conductas de agresión de unos alumnos hacia otros.

La técnica aplicada en la intervención ha sido concretamente el Jigsaw, una técnica que posibilita un contacto directo y personal entre todos los alumnos del aula. Este contacto directo ha reducido la percepción de amenaza y la ansiedad intergrupal, lo que a su vez ha disminuido las agresiones y ha facilitado que los alumnos testigos de ellas, aprecien un descenso de las mismas. Como afirman Ramírez \& Rodríguez (2006), el hecho de que los alumnos estén en el aula, y por tanto exista solo un contacto presencial, no garantiza una mejora de las relaciones intergrupales. Distintos estudios (Cook, 1985; León, Felipe, Iglesias \& Latas, 2011; León, Gozalo y Polo, 2012; Stephan, 1987) demuestran que para que el contacto sea efectivo debe tratarse de un contacto directo y personal que facilite la percepción de las características individuales de los demás. La mejora en la convivencia se puede lograr, como afirma Brown (1988), mediante contactos en condiciones de igualdad y buscando objetivos comunes mediante la cooperación. En definitiva, los alumnos participantes de esta investigación se han beneficiado de un contacto directo y personal, motivado por las técnicas de aprendizaje cooperativo, que han mejorado sus relaciones.

Por último, las situaciones de aprendizaje cooperativo han aumentado la habilidad para ponerse en la perspectiva emocional de los otros. En palabras de Aronson \& Osherow (1980), la técnica Jigsaw incrementa la atracción de los estudiantes hacia sus compañeros, disminuye la competitividad y aumenta la capacidad para ponerse en el lugar o papel de otra persona (Moriña, 2011). La empatía nos hace ser más 
sensibles a las necesidades y demandas de nuestros compañeros.

Siendo el acoso escolar un fenómeno de carácter grupal, parte de las intervenciones tienen que ir dirigidas al grupo-aula y a su funcionamiento. En este sentido, y así lo demuestran los resultados, la metodología en aprendizaje cooperativo es eficaz en su intervención sobre los agresores y las víctimas, y también sobre aquellos alumnos, que no participan directamente de la situación de acoso escolar, pero juegan un papel importante en el origen y mantenimiento de los episodios de malos tratos, tanto favoreciendo como atajando el problema. Intervenir sobre el conjunto de la clase, ayuda a prevenir el acoso escolar y a favorecer interacciones sociales entre compañeros, que inhiben su aparición (DíazAguado, 2005; Vega, Vidal, \& García, 2013).

En líneas de investigación con proyección al futuro, se debería hacer un análisis más exhaustivo, dentro de los grupos de aprendizaje cooperativo, del proceso interactivo entre los alumnos en el aula. Igualmente, habría que determinar qué técnica de aprendizaje cooperativo es más eficaz para propiciar un cambio que disminuya la violencia. En este estudio, se utiliza para la intervención en el aula, la técnica de Jigsaw. Algunas investigaciones como las de Slavin (1983) obtienen resultados más positivos con las técnicas de aprendizajes por equipos (Student Team Learning) que con las técnicas de aprendiendo juntos (Learning Together). Este hecho es confirmado por DíazAguado (1996), para quien los métodos más eficaces para favorecer el desarrollo de la tolerancia, en contextos heterogéneos, son los TGT (Torneos de Equipos y Juegos) y los STAD (División de los Estudiantes por Equipos de Rendimiento). Ambos métodos pertenecen a las técnicas de aprendizaje por equipos. Otros estudios han demostrado la eficacia del método Jigsaw (Llorent \& Varo, 2013; Walker \&Crogan, 1998). Sin duda alguna, esta es una cuestión que habrá que confirmar en investigaciones futuras.

Partiendo de la idea de que la metodología de aprendizaje cooperativo despierta en los alumnos interés y preocupación de unos hacia otros (Pedreira \& González-Fontao, 2014; Slavin, 1992), se trataría de determinar de qué manera las técnicas de aprendizaje influyen en el alumnado observador, para mantenerse no solo como espectador pasivo de lo que ocurre a su alrededor, detectando cambios en las interacciones entre sus compañeros, sino también para transformarse en espectador que denuncie las situaciones injustas y violentas (Tresgallo, 2008).

Un planteamiento de futuro consiste en elaborar un instrumento, que nos ayude a recoger información acerca de si el trabajo en el aula, desde la metodología de aprendizaje cooperativo influye en el observador, para no mantenerse como mero testigo de las conductas propias de acoso escolar, sino también, para intervenir de alguna manera en la reducción de las mismas.

Por último, el factor tiempo es relevante en este tipo de intervenciones, ya que si en cuatro meses de aplicación de técnicas de aprendizaje cooperativo en el aula, se han obtenido resultados positivos en relación con ciertas conductas de agresión (físicas indirectas y de exclusión social), una intervención más duradera puede llevar a mejores resultados, en las distintas modalidades de agresión, produciendo cambios tanto a nivel conductual como actitudinal. El aprendizaje cooperativo es una metodología que genera actitudes más positivas hacia los demás (Pedreira \& González-Fontao, 2014) y aunque las actitudes poseen una naturaleza relativamente estable y duradera, es cierto que pueden modificarse puesto que son adquiridas, y por tanto, susceptibles de ser fomentadas, reorientadas o incluso cambiadas, es decir, enseñadas (Eagly \& Chaiken, 1993), por tanto, una intervención en aprendizaje cooperativo sostenida en el tiempo, puede dar lugar a un cambio de actitudes en el conjunto de la clase, teniendo efectos significativos sobre la conducta agresiva después de transcurrido bastante tiempo. 


\section{Referencias}

Antolín, A., Martín-Pérez, G., \& Barbá, J. (2012). El aprendizaje cooperativo para la mejora de la socialización y la educación a través del conflicto. La Peonza, Revista de Educación Física para la Paz, 7, 3-11. Recuperado de https://dialnet.unirioja.es/se rvlet/articulo? codigo $=3907247$

Aronson, E., \& Osherow, N. (1980). Cooperation, prosocial behaviour, and academic performance. En L. Bickman (Ed.), Applied social psychology annual, Vol. 1 (pp. 163-196). Beverly Hills: Sage.

Avilés, J. M., \& Monjas, I. (2005). Estudio de incidencia de la intimidación y el maltrato entre iguales en la educación secundaria obligatoria mediante el cuestionario CIMEI (Avilés, 1999)-Cuestionario sobre Intimidación y Maltrato Entre Iguales. Anales de Psicología, 21, 27-41.

Avilés, J. M. (2004) ¿Cómo abordar un caso de bullying? En R. Belandia (coord.) IV Curso de verano. Prevención de riesgos laborales en la enseñanza, (pp. 143-173). Bilbao: Instituto Vasco de Ergonomía y Steeeilas.

Avilés, J.M., Irurtia, M.J., García-López, L.J., \& Caballo, V. (2011). El maltrato entre iguales: "Bullying". Psicología Conductual, 19 (1), 57-90. Recuperado de http://www.funveca.org/revista/pedidos/ product.php?id_product $=467$

Brown, R. (1988). Group processes. Dynamics within and between groups. Oxford: Blackwell.

Caballo, V., Calderero, M., Arias, B., Salazar, I. C., \& Irurtia. M. J. (2012). Desarrollo y validación de una nueva medida de auto informe para evaluar el acoso escolar (Bullying). Psicología Conductual, 20(3), 625-647. Recuperado de https://www.researchgate.net/profile/Vi cente_Caballo/publication/259481844_De velopment_and_validation_of_a_new_self -report_assessment_measure_of_Desarrollo_y_validacion_de_una_nueva medida_de_autoinforme_para_evaluar_el_ acoso_escolar_bullying/links/0deec52c1c4 40ee $\overline{12} 80000 \overline{0}$.pdf

Cerezo, F. (2014). Diferencias en estatus social entre roles en "Bullying": Un análisis sociométrico. Bordón. Revista de Pedagogía, 66(2), 29-42. Recuperado de https://dialnet.unirioja.es/servlet/articul o? codigo $=4673867$

Cerezo, F. (2006). Violencia y victimización entre iguales. El bullying: estrategias de identificación y elementos para la intervención a través del Test BullS. Revista Electrónica de Investigación Psicoeducativa, 4(2), 333-352. Recuperado de http://www.sinohacesnadasosparte.org/ Download/spanish/02_BULLYING_EN_E SPANOL.pdf

Cerezo, F., \& Sánchez, C. (2013). Eficacia del programa CIP para la mejora de la convivencia escolar y la prevención del bullying en alumnos de Educación Primaria. Apuntes de Psicología, 31(2), 173-181. Recuperado de http://www.apuntesdepsico logia.es/index.php/revista/article/view/320

Cohen, J. (1977). Statistical power analysis for the behavioral sciences. Nueva York: Academic Press.

Cook, S. W. (1978). Interpersonal and attitudinal outcomes in cooperating interracial groups. Journal of Research and Development in Education, 12, 27-113.

Cook, S. W. (1985). Experimenting on social issues: The case of school desegregation. American Psychologist, 40, 452-460.

Cuevas, M. C., \& Marmolejo, M. A. (2016). Observadores: un rol determinante en el acoso escolar. Pensamiento Psicológico, 14(1), 89-102. http://dx.doi.org/10.11144/Javeria nacali.PPSI14-1.orda

Defensor del Pueblo (2006). Violencia escolar: el maltrato entre iguales en la Educación Secundaria Obligatoria (1999-2006). Madrid: Publicaciones del Defensor del Pueblo.

Del Barrio, C., Martín, E., Montero, I., Fernández I., \& Gutiérrez, H. (2003). La realidad del maltrato entre iguales en los centros de secundaria españoles. Infancia 
Maa Isabel Polo del Río, Santiago Mendo Lázaro, Fernando Fajardo Bullón, Et al.

y Aprendizaje, 26, 25-47. Recuperado de http://www.unav.edu/matrimonioyfamil ia/observatorio/indexbase.php? $\mathrm{cmd}=$ searc h4\&id $=24946$

Devries, D.L \& Edwards, K.J. (1974). Learning games and student teams: Their effects on classroom process. American Educational Research Journal, 4, 307-318.

Díaz-Aguado, M. J. (2005). Por qué se produce la violencia escolar y cómo prevenirla. Revista Iberoamericana de Educación, 37, 17-47. Recuperado de http://www.redalyc.org/pdf/ 800/80003703.pdf

Díaz-Aguado, M. J. (2003). Educación intercultural y aprendizaje cooperativo. Madrid: Pirámide.

Díaz-Aguado, M.J. (1996) Escuela y tolerancia. Madrid: Pirámide.

Eagly, A. H., \& Chaiken, S. (1993). The psychology of attitudes. Fort Worth, TX: Harcourt, Brace, Jovanovich.

Farrington, D. P. (1993). Understanding and preventing bullying. Crime and Justice: Review of Research, 17, 381-458.

Goikoetxea, E., \& Pascual, G. (2002). Aprendizaje Cooperativo: Bases teóricas y hallazgos empíricos que explican su eficacia. Educación XX1, 5, 227-247. Recuperado de http://revistas.uned.es/index.php/educa cionXX1/article/viewFile/392/342

Gutiérrez, R., Benítez, J. L., Machado, C., \& Justicia, F. (2012). Estudio de las atribuciones hacia el maltrato entre iguales del alumnado agresor frente al no agresor, mediante el Cuestionario SCAN-Bullying. Electronic Journal of Research in Educational Psychology, 10(2), 545-568. Recuperado de http://www.investigacion-psicopedagogi ca.org/revista/articulos/27/english/Art_27_ 669.pdf

Haro, I., \& García, B. (2014). Variables emocionales y socio morales asociadas con el tipo de rol que asumen los alumnos y alumnas en el maltrato entre iguales. Apuntes de Psicología, 32 (1), 15-23. Recuperado de http://www.apuntesdepsico logia.es/index.php/revista/article/view/482
Hoyos, O. L., Llanos, M., \& Valega, S. (2012). El maltrato entre iguales por abuso de poder en el contexto universitario: incidencia, manifestaciones y estrategias de solución. Universitas Psychologica, 11(3), 793-802. Recuperado de http://revistas.javeriana.edu.co/index.p hp/revPsycho/article/viewFile/793/2872

Johnson, D. W. (1975). Cooperativeness and social perspective taking. Journal of Personality and Social Psychology, 312. 241-244.

Johnson, D.W., Johnson, R. T., \& Holubec, E. J. (1999). El aprendizaje cooperativo en el aula. Buenos Aires: Paidós.

Johnson, D.W. \& Johnson, R.T. (1990). Social skills for successful group work. Educational Leadership, 47, 29-33.

Johnson, D.W., \& Johnson, R.T. (2000). The three $\mathrm{Cs}$ of reducing prejudice and discrimination. In S. Oskamp (Ed.), Reducing prejudice and discrimination (pp. 239-268). Mahwah, NJ: Lawrence Erlbaum Associates Publishers.

Johnson, D.W., Johnson, R., \& Maruyama, G. (1983). Interdependence and interpersonal attraction among heterogeneous and homogeneous individuals. A theoretical formulation and meta-analysis of the research. Review Educational Research, 53, 5-54. http://dx.doi.org/10.3102/003465430 53001005

Kirk, R. E. (1996). Practical significance: a concept whose time has come. Educational and Psychological Measurement, 56, 746-75.

León, B., Mendo, S., Felipe, E., Polo, M. I., \& Fajardo, F. (2017). Potencia de equipo y aprendizaje cooperativo en el ámbito universitario. Revista de Psicodidáctica, 22(1). Recuperado de http://www.elsevier.es/es-revista-revista -psicodidactica-243-articulo-potencia-equi po-aprendizaje-cooperativo-el-X11361034 16605332

León, B., Polo, M. I., Gozalo, M., \& Mendo, S. (2016). Relevancia del aprendizaje cooperativo sobre los diferentes perfiles de la dinámica bullying. Un análisis mediante 
pruebas de tamaño del efecto. Anales de Psicología, 32 (1), 80-88. http://dx.doi.org/1 0.6018/analesps.32.1.183141

León, B., Felipe, E., Mendo, S., \& Iglesias, D. (2015). Habilidades sociales en equipos de aprendizaje cooperativo en el contexto universitario. Psicología Conductual, 23(2), 191-214. Recuperado de https://dialnet.uni rioja.es/servlet/articulo?codigo $=5186155$

León, B., Gozalo, M., \& Polo, M. I. (2012). Aprendizaje cooperativo y acoso entre iguales. Infancia y Aprendizaje, 35(1), 23-35. http://dx.doi.org/10.1174/02103701 2798977494

León, B., Felipe, E., Gómez, T., \& López, V. (2011). Acoso Escolar en la Comunidad de Extremadura vs. Informe español del Defensor del Pueblo (2006). Electronic Journal of Research in Educational Psychology, 9(2), 565-586. Recuperado de http://www.investigacion-psicopedagogi ca.org/revista/articulos/24/english/Art_24_ 564.pdf

León, B., Felipe, E., Iglesias, D., \& Latas, C. (2011). El aprendizaje cooperativo en la formación inicial del profesorado de Educación Secundaria. Revista de Educación, 354, 715-729. Recuperado de ht tp://adicodeporte.es/adicode/images/stories /articulos/articulos-p-deliberada/revista_de educacion.pdf

León, B., Gozalo, M. \& Vicente, F. (2004). Factores mediadores en el aprendizaje cooperativo: los estilos de conducta interpersonal. Apuntes de Psicología, 22(1), 61-74. Recuperado de http://www.apuntesdepsicologia.es/inde x.php/revista/article/view/41

Llorent, V.J., \& Varo, J.C. (2013). Innovaciones didácticas para fomentar el aprendizaje y la cohesión social en el aula. La técnica puzle con equipo de sabios. Innovación Educativa, 23, 179-196. Recuperado de http://www.usc.es/revistas/index.php/ie/ article/viewFile/711/1490

López, V., Bilbao, M. A., \& Rodríguez, J. I. (2012). La sala de clases sí importa: incidencia del clima de aula sobre la percepción de intimidación y victimización entre escolares. Universitas Psychologica, 11(1), 91-101. Recuperado de https://www.google.com.co/url?sa $=t \& r$ $\mathrm{ct}=\mathrm{j} \& \mathrm{q}=$ \&esrc $=\mathrm{s} \&$ source $=$ web\&cd $=1$ $\&$ cad $=$ rja\&uact $=8 \&$ ved $=0$ ahUKEwju-o bFqfPSAhXI5CYKHRPCC5AQFggaMA A\&url $=$ http\%3A\%2F\%2Frevistas.javeria na.edu.co\%2Findex.php\%2FrevPsycho\%2 Farticle\%2Fdownload\%2F1002\%2F1622\& usg $=$ AFQjCNGCtZAoA9npd5MTF9pKv 29 gOXySQ\&sig2 =WB6NOb-_cTMleKb OGAUzDA

Mendoza, B., Morales, T., \& Arriaga, Y. (2015). Variables Proximales relacionados con Violencia Escolar y Bullying en alumnado de bachillerato. Psychology, Society $\mathbb{E}$ Education, 7(2), 185-200. Recuperado de h ttp://www.psye.org/articulos/Mendoza.pdf

Molina, J. A., \& Vecina, P. (2015). Bullying, ciberbullying y sexting. ¿Cómo actuar ante una situación de acoso? Madrid: Ediciones Pirámide.

Moriña, A. (2011). Aprendizaje cooperativo para una educación inclusiva: desarrollo del programa PAC en un aula de Educación Primaria. ESE, Estudios Sobre Educación, 21, 199-216. Recuperado de https://www.google.com.co/url?sa $=$ t\&r $\mathrm{ct}=\mathrm{j} \& \mathrm{q}=\&$ \&esrc $=\mathrm{s} \&$ source $=$ web\&cd $=1$ $\&$ cad $=$ rja\&uact $=8 \&$ ved $=0$ ahUKEwi3rc Dur_PSAhWCKyYKHZc8A7IQFggaMA A\&url $=$ https\%3A\%2F\%2Fdialnet.unirioj a.es $\% 2$ Fservlet $\% 2$ Farticulo\%3Fcodigo $\% 3$ D3791344\&usg =AFQjCNHY2fWclXWr vb0cT81AfvVvLNm2uA\&sig2=AoDbaM 19NSLBDFzoVMvPxw

Olweus, D. (1993). Bullying at school: What we know and what we can do.Oxford: Blackwells.

Olweus, D. (1983). Low school achievement and agressive behaviour in adolescent boys. In D. Magnusson \& V. Allen (Eds.), Human developrnent. An interactional perspective (pp. 353-365). Nueva York: Academic Press.

Ortega, R., Del Rey, R., \& Casas, J. A. (2013). La convivencia escolar: clave en la predicción del bullying. Revista 
Maa Isabel Polo del Río, Santiago Mendo Lázaro, Fernando Fajardo Bullón, Et al.

Iberoamericana de Evaluación Educativa, 6(2), 91-102. Recuperado de https://dialne t.unirioja.es/descarga/articulo/4697267.pdf

Ovejero, A. (1990). El aprendizaje cooperativo. Barcelona: PPU.

Pedreira, M. P., \& González-Fontao, M. P. (2014). El aprendizaje cooperativo en un centro de primaria: una experiencia inclusiva. Innovación educativa, 24, 259-272. Recuperado de https://www.google.com.co/url?sa $=$ t\&r $\mathrm{ct}=\mathrm{j} \& \mathrm{q}=\&$ \&esrc $=\mathrm{s} \&$ source $=\mathrm{web} \& \mathrm{~cd}=1$ $\&$ cad $=$ rja\&uact $=8 \&$ ved $=0$ ahUKEwjjtc YqPPSAhUI2yYKHYuUCLoQFggYMAA \&url =http $\% 3 \mathrm{~A} \% 2 \mathrm{~F} \% 2 \mathrm{Fwww}$.usc.es $\% 2 \mathrm{Fr}$ evistas\%2Findex.php\%2Fie\%2Farticle\%2F download\%2F1671\%2F2405\&usg=AFQj CNG6Xm_yLTv5yVJFRXFz1azOP8uI7A \&sig2 = LhyYGO7U3RSDje-AGWFIIA\&b $\mathrm{vm}=$ bv.150729734,d.eWE

Pérez-Sánchez, A. M., \& Poveda, P. (2008). Efectos del aprendizaje cooperativo en la adaptación escolar. Revista de Investigación Educativa, 26(1) 73-94. Recuperado de http://www.redalyc.org/pdf/2833/28332 1884005.pdf

Polo, M. I., León, B., Felipe, E., Fajardo, F., Gómez, T., \& Mendo, S. (2015). Análisis de la Socialización sobre Perfiles de la dinámica bullying. Universitas Psychologica, 14(3), 1117-1128. http://dx.doi.org/10.111 44/Javeriana.upsy14-3.aspd

Ramírez-Pavelic, M. (2013). Aprendizaje cooperativo: un modelo a aplicar frente al maltrato entre iguales. Liberabit, 19(1), 93-99. Recuperado de http://www.scielo.org.pe/scielo.php?pid $=$ S1729-48272013000100009\&script $=$ sci arttext

Ramírez, M. C., \& Rodríguez, A. (2006). Variables predictoras de la actitud hacia los inmigrantes en la Región de Murcia (España). Anales de Psicología, 22, 76-80.

Ruíz, R., Riuró, M., \& Tesouro, M. (2014). Estudio del bullying en el ciclo superior de primaria. Educación XX1, 18(1), 345-368. Recuperado de http://www.redalyc.org/pdf/ 706/70632585015.pdf
Slavin, R. E. (1978). Student teams and comparison among equals: effects on academic performance and student attitudes. Journal of Educational Psychology, 70(4), 532-538. Recuperado de http://psycnet.apa.org/index.cfm?fa $=$ bu y.optionToBuy\&id=1979-24669-001

Slavin, R. E. (1983). Cooperative Learning. New York: Longman

Slavin, R. E. (1992). Aprendizaje cooperativo. En C. Rogers \& P. Kutnick (Eds.), Psicología Social de la Escuela Primaria (pp. 247-270). Barcelona: Paidós.

Slavin, R. E., \& Cooper, R. (1999). Improving intergroup relations: lessons learned from cooperative learning programs. Journal of Social Issues, 55(4), 647-663. http://dx.doi. org/10.1111/0022-4537.00140

Smith, P. K., Boulton, M. J., \& Cowie, H. (1993). The impact of cooperative group work on ethnic relations in middle school. School Psychology International, 14, 21-42. Recuperado de http://journals.sagepub.com /doi/abs/10.1177/0143034393141002

Stephan, W. (1987). The contact hypothesis in intergroup relations. Review of Personality and Social Psychology, 9, 13-40.

Trautmann, A. (2008). Maltrato entre pares o "bullying". Una visión actual. Revista Chilena de Pediatría, 79(1), 13-20. http://dx.doi.org/10.4067/S0370-41 062008000100002

Tresgallo, E. (2008). Violencia escolar ("Bullying"): documento para padres y educadores. Revista española de Orientación y Psicopedagogía, 19(3), 328-333. Recuperado de http://www.redaly c.org/pdf/3382/338230780007.pdf

Trianes, M. V., Muñoz, A., \& Jiménez (2007). Las relaciones sociales en la infancia y la adolescencia y sus problemas. Madrid: Pirámide.

Vega, M. L. Vidal, D., \& García, M. P. (2013). Avances acerca de los efectos del aprendizaje cooperativo sobre el logro académico y las habilidades sociales en relación con el estilo cognitivo. Revista Colombiana de Educación, 64, 
155-174. http://dx.doi.org/10.17227/01203 916.64rce155.174

Walker, I. \& Crogan, M. (1998). Academic performance, prejudice, and the jigsaw classroom: New pieces to the puzzle. Journal of Community and Applied Social Psychology, 8, 381-393.

\section{Notas}

* Artículo de investigación. 